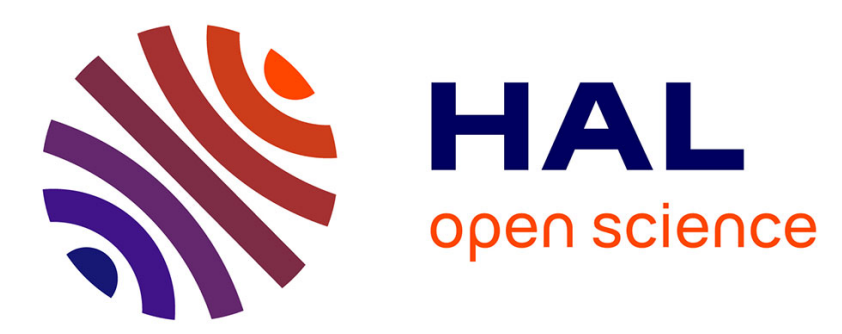

\title{
La modélisation en équilibre général dynamique et stochastique des cycles économiques en Afrique Sub-saharienne: une revue de la littérature
}

Claude Francis Naoussi, Fabien Tripier

\section{- To cite this version:}

Claude Francis Naoussi, Fabien Tripier. La modélisation en équilibre général dynamique et stochastique des cycles économiques en Afrique Sub-saharienne: une revue de la littérature. 2012. hal$00733393 \mathrm{v} 3$

\section{HAL Id: hal-00733393 \\ https://hal.science/hal-00733393v3}

Preprint submitted on 30 Nov 2012

HAL is a multi-disciplinary open access archive for the deposit and dissemination of scientific research documents, whether they are published or not. The documents may come from teaching and research institutions in France or abroad, or from public or private research centers.
L'archive ouverte pluridisciplinaire HAL, est destinée au dépôt et à la diffusion de documents scientifiques de niveau recherche, publiés ou non, émanant des établissements d'enseignement et de recherche français ou étrangers, des laboratoires publics ou privés. 
EA 4272

\section{La modélisation en équilibre général dynamique et stochastique des cycles économiques en Afrique Sub-saharienne : une revue de la littérature}

\section{Claude Francis Naoussi * Fabien Tripier **}

$2012 / 40$

* Université de Nantes, LEMNA - France

** Université de Lille 1, CLERSE \& CEPII - France

\section{Laboratoire d'Economie et de Management Nantes-Atlantique} Université de Nantes 


\title{
La modélisation en équilibre général dynamique et stochastique des cycles économiques en Afrique Sub-saharienne : une revue de la littérature ${ }^{1}$
}

\author{
Claude Francis Naoussi (Université de Nantes, Lemna)² \\ Fabien Tripier (Université de Lille 1, Clersé \& CEPII) 3
}

\footnotetext{
${ }^{1}$ Nous remercions la Chaire Finance de la Fondation de projets de l'Université de Nantes pour son soutien financier.

2 claude.naoussi-defounkou@univ-nantes.fr

${ }^{3}$ fabien.tripier@univ-lille1.fr. CLERSE, Université de Lille 1 - Bâtiment SH2, 59655 Villeneuve d'Ascq Cedex
} 
La modélisation en équilibre général dynamique et stochastique des cycles économiques en Afrique Sub-saharienne : une revue de la littérature

Résumé. Cet article propose une revue de la littérature sur les modèles d'équilibre général dynamique et stochastique appliqués aux pays d'Afrique Sub-saharienne. Cette littérature a pour double objectif (i) d'identifier les facteurs spécifiques à ces économies susceptibles d'expliquer leur très forte instabilité et (ii) d'évaluer les politiques monétaires et fiscales adéquates face à ces facteurs. Nous présentons les avancées de cette littérature à l'aune de ces deux objectifs et en soulignons les limites.

Mots-clefs : Développement ; Cycle ; Afrique ; Équilibre général ; DSGE JEL Classification : E32, F41, O55

\section{Dynamic and Stochatic General Equilibrium Models for Sub-Saharan Africa Business Cycles: a Review}

Abstract. This article reviews the recent literature on Dynamic and Stochastic General Equilibrium Models for Sub-Saharan Africa business cycles. This literature has two objectives (i) to identify the factors specific to these countries that explain their high volatility and (ii) to assess the relevant monetary and fiscal policies given these factors. We present the progress made by this literature and underscore its limits.

Keywords: Development ;Cycle ;Africa ;General equilibrium, DSGE JEL Classification: E32, F41, O55 


\section{La modélisation en équilibre général dynamique et stochastique des cycles économiques en Afrique Sub-saharienne : une revue de la littérature}

\section{Introduction}

Cet article propose une revue de la littérature consacrée aux cycles économiques en Afrique Sub-saharienne (ASS) basée sur la modélisation en équilibre général dynamique et stochastique. Deux motivations fondent cette littérature : (i) le coût des cycles économiques pour les économies africaines et (ii) le progrès méthodologique qu'a apporté la modélisation en équilibre général dynamique et stochastique pour l'analyse du cycle économique.

\subsection{Le coût des cycles économiques en ASS}

La lutte contre la pauvreté est la première priorité des Etats d'ASS et des institutions internationales. Les politiques mises en œuvre visent à promouvoir la croissance économique, afin d'élever le niveau moyen de richesse par habitant dans ces pays, ainsi qu'à appréhender les différentes dimensions de cette pauvreté comme en témoignent les objectifs du millénaire et le développement des indicateurs de développement humain.

Les économies d'ASS souffrent de niveaux très faibles de développement humain, mais elles sont également caractérisées par une grande instabilité ${ }^{4}$ qui n'est pas explicitement intégrée dans la définition de ces politiques. La stabilité ne fait pas partie des objectifs du millénaire et n'est pas non plus prise en compte dans le calcul

4 Ramey et Ramey (1995) ont réalisé une comparaison internationale des indicateurs de croissance et d'instabilité et montré qu'une faible croissance économique, à l'origine d'une stagnation du revenu moyen par habitant, s'accompagne de manière significative d'une plus forte volatilité de l'économie. Rand et Tarp (2002) comparent les cycles économiques au niveau international et montrent qu'ils sont plus courts et d'une plus grande amplitude dans les pays en développement. 
des indicateurs de développement humain ${ }^{5}$. Or, les recherches récentes suggèrent que cette instabilité peut représenter un coût majeur en termes de bien-être.

Du point de vue du bien-être individuel, la volatilité des revenus et de la consommation constitue une perte de bien-être au même titre qu'une consommation insuffisante en quantité et trop faiblement croissante au cours du temps. Dans la lignée de Lucas (1987), il est devenu courant d'utiliser ce critère de bien-être individuel pour quantifier et comparer la contribution de ses différentes composantes : le niveau stationnaire de la consommation, sa croissance de longterme et ses fluctuations. La principale conclusion de Lucas (1987) pour les EtatsUnis est que les fluctuations de la consommation contribuent peu au bien-être individuel par rapport aux autres composantes, notamment la croissance de long terme. Pour l'auteur, ce résultat minore considérablement l'intérêt des politiques de stabilisation macroéconomique et pourrait justifier leur absence dans les objectifs de politiques économiques des pays en développement. Néanmoins, Pallage et Robe (2003) ont montré que le coût en bien-être des fluctuations varie considérablement selon le niveau de développement. II est d'autant plus élevé que l'instabilité économique est forte et la croissance faible ; conditions qui sont précisément réunies pour les pays d'ASS où ce coût peut atteindre des niveaux très importants (vingt fois supérieur à celui calculé pour les États-Unis selon Pallage et Robe (2003)).

L'importance de la question de la stabilité en ASS est encore renforcée si on ajoute à ce coût direct des fluctuations sur le bien-être, leurs coûts indirects. Les coûts indirects des fluctuations correspondent à leurs effets négatifs sur les autres composantes du bien-être individuel: la consommation d'état stationnaire et la croissance de long terme. L'instabilité des économies d'ASS peut effectivement pénaliser les investissements en capital humain et physique et être ainsi à l'origine des faibles niveaux de production et de croissance observés; voir Collier et Gumming (1999), Turnovsky et Chattopadhyay (2003) et Loayza et al. (2007) pour des synthèses des résultats empiriques et des principaux mécanismes en jeu.

5 Les objectifs du millénaire définis en 2000 au sein de l'Organisation des Nations Unis portent sur la pauvreté, mais également sur la nutrition, l'accès à l'éducation, la santé, l'égalité et l'environnement. L'intégration des multiples facettes du développement humain a été concrétisée en 1990 par la création d'indicateurs du développement humain dans le cadre du Programme des Nations Unis pour le Développement. 


\subsection{La modélisation des cycles économiques en équilibre général}

La modélisation des cycles économiques en équilibre général s'est imposée suite aux critiques adressées par Lucas (1976) et Kydland et Prescott (1977) aux modèles macroéconomiques dénués de fondements microéconomiques. La prise en compte de la réaction des agents économiques aux modifications de leur environnement économique est aujourd'hui considérée comme indispensable pour une évaluation cohérente des politiques économiques ${ }^{6}$. Le succès des modèles d'équilibre général dynamique et stochastique (DSGE ${ }^{7}$ ) pour l'étude des politiques conjoncturelles s'explique par leur capacité à intégrer cette réaction des agents économiques à la politique économique en présence d'une incertitude macroéconomique et à rendre compte des principaux faits du cycle économique ${ }^{8}$.

Ces modèles ont connu d'importantes évolutions depuis la contribution pionnière de Kydland et Prescott (1982). La première porte sur la spécification de ces modèles. D'abord développés dans une perspective néoclassique de perfection des marchés et d'inutilité des politiques conjoncturelles, ils ont été étendus à la présence d'imperfections de marché légitimant des politiques conjoncturelles actives ; voir notamment Woodford (2003). La seconde porte sur le domaine d'application de ces modèles. Initialement développés pour l'économie américaine, ces modèles ont été très largement appliqués aux autres économies les plus avancées - voir Danthine et Donaldson (1993) pour l'Europe - ainsi qu'aux pays émergents. La coïncidence d'une forte croissance et d'importantes crises économiques et financières au sein des pays émergents a motivé une très importante littérature ${ }^{9}$ mobilisant ces modèles DSGE afin de comprendre l'origine de ces crises et de définir les meilleures politiques économiques pour ces pays. En comparaison aux pays émergents, les

6 Acemoglu (2010) propose une discussion plus large montrant l'importance de l'analyse en équilibre général pour la définition des politiques de développement.

7 Pour "Dynamic and Stochastic General Equilibrium".

8 Dans l'article du New Palgrave Dictionnary of Economics, Bénassy (2008) avance trois raisons pour expliquer le succès de cette modélisation : (i) l'existence de fondements microéconomiques solides; (ii) sa capacité à intégrer dans un cadre homogène des approches différentes de l'équilibre macroéconomique; et (iii) son pouvoir explicatif des faits du cycle économique. Stadler (1994) et Hairault (1999) ont proposé des premières revues de la littérature sur cette modélisation.

9 Mendoza et Arellano (2002) proposent une synthèse de cette littérature. 
applications aux pays les plus pauvres, notamment d'ASS restent limitées. L'apport de ces applications et les perspectives de développement de cette littérature constituent l'objet de cette revue de la littérature.

\subsection{Les objectifs des modèles DSGE appliqués aux pays d'ASS}

Si l'on admet (i) le coût important que représentent les fortes fluctuations macroéconomiques dans les pays d'ASS et (ii) la nécessité de se placer dans des cadres d'équilibre général pour comprendre leur origine et définir des politiques économiques adéquates ; le développement de modèles DSGE pour l'ASS apparaît comme naturel. Nous organisons la présentation de ces modèles autour des deux objectifs de cette littérature.

Le premier objectif est d'identifier les sources d'impulsion et de propagation spécifiques à ces pays pouvant expliquer l'ampleur de leurs fluctuations macroéconomiques $^{10}$. Les contributions de Mendoza (1995), Kose et Reizman (2001), Kose (2002) et Arellano et al. (2009) mettent en avant les conséquences de chocs spécifiques à ces pays portant sur les termes de l'échange, les prix relatifs internationaux, le taux d'intérêt et l'aide au développement. Özbilgin (2010) considère que la participation limitée des agents aux marchés financiers est le mécanisme d'amplification spécifique à ces pays susceptible d'expliquer l'ampleur de leurs fluctuations.

Le second objectif est de définir les politiques conjoncturelles tenant compte des spécificités de ces pays. La politique monétaire a été plus particulièrement étudiée par Peiris et Saxegaard (2010) pour la Mozambique et Houssa et al. (2010) pour le Ghana. Les contributions d'Adam et al. (2009) et Dagher et al. (2010) intègrent dans la définition de la politique optimale des chocs spécifiques à ces pays concernant l'aide internationale et les prix du pétrole.

10 Les modèles DSGE s'inscrivent généralement dans le schéma impulsion-propagation. Les impulsions correspondent aux chocs exogènes frappant l'économie qui sont à l'origine du cycle économique. Les mécanismes de propagation déterminent la façon dont ces chocs sont transmis et propagés dans l'économie et sont eux-mêmes déterminés par le fonctionnement des marchés et le comportement des agents.. 
Nous consacrons dans la suite les sections 2 et 3 à ces deux champs de la littérature, puis proposons en conclusion dans la section 4 un bilan critique de cette littérature.

\section{Les origines des fluctuations en ASS}

Dans le schéma impulsion-propagation, la forte volatilité macroéconomique spécifique aux pays d'ASS s'explique soit par des chocs (ou impulsions) spécifiques à ces économies soit par le fait que les effets des chocs (par ailleurs identiques à ceux d'autres pays) y sont amplifiés et propagés par des mécanismes spécifiques.

\subsection{Les chocs sur les termes de l'échange [Mendoza (1995)]}

Mendoza (1995) est le premier à appliquer un modèle DSGE à des économies d'ASS. II considère plus précisément deux groupes de pays : le premier est composé de sept pays industrialisés et le second de vingt-quatre pays en développement dont cinq d'ASS. Plus que la différence entre ces deux catégories de pays, Mendoza (1995) cherche avant tout à expliquer pourquoi les conditions de parité des pouvoirs d'achat et de taux d'intérêt ne sont pas vérifiées au cours du cycle.

Le modèle utilisé par Mendoza (1995) est celui d'une petite économie ouverte dans laquelle le ménage représentatif de cette économie a une durée de vie illimitée et consomme quatre types de biens : les biens non échangeables ( $n$ ), les biens exportables $(\mathrm{x})$, les biens importables (f) et le loisir (l). Les préférences du ménage sont décrites par les équations suivantes :

$$
\begin{aligned}
& U(x, f, l)=E \llbracket \sum_{t=0}^{\infty}\left\{u\left(x_{t}, f_{t}, n_{t}, l_{t}\right) \cdot \exp \left(-\sum_{\tau}^{t-1} v\left(x_{\tau} f_{\tau} n_{\tau} l_{\tau}\right)\right\} \rrbracket\right. \\
& u(x, f, n, l)=\frac{\left(\left[\left(x^{a} f^{1-a}\right)^{-\mu}+n^{-\mu}\right]^{-\frac{1}{\mu}} l^{\omega}\right)^{1-\gamma}}{1-\gamma} \\
& \left.v(x, f, n, l)=\beta \ln \left(\left(x^{a} f^{1-a}\right)^{-\mu}+n^{-\mu}\right]^{-\frac{1}{\mu}} l^{\omega}\right) \\
& \quad 0 \leq a \leq 1, \quad \mu>-1, \quad \gamma>1, \quad \beta>0, \quad \omega>0
\end{aligned}
$$


où $1 /(1+\mu)$ est le taux de substitution constant entre les biens échangeables $(x$ et $f)$ et non échangeables, a est la part des biens exportables allouée aux dépenses échangeables, $\omega$ est le coefficient d'élasticité de l'offre du travail et $\beta$ est le coefficient de préférence intertemporelle. La contrainte budgétaire de l'économie est décrite par les équations suivantes :

$$
\begin{aligned}
& f_{t}+e_{t}^{p} p^{x} x_{t}=Q\left(e_{t}^{p} p^{x} e_{t}^{x}\left(K_{t}^{x}\right)^{1-\alpha x}\left(L^{x}\right)^{\alpha x}+e_{t}^{f}\left(K_{t}^{f}\right)^{1-\alpha f}\left(L^{f}\right)^{\alpha f}\right)-K_{t+1}+K_{t}(1-\delta)- \\
& \frac{\emptyset}{2}\left(K_{t+1}-K_{t}\right)^{2}-A_{t+1}+A_{t}\left(1+r^{*}\right) \\
& \quad n_{t}=Q e_{t}^{n}\left(K^{n}\right)^{1-\alpha n}\left(L_{t}^{n}\right)^{\alpha n}-K^{n}+K^{n}(1-\delta) \\
& \quad l_{t}+L_{t}^{n}+L^{x}+L^{f}=T
\end{aligned}
$$

Les firmes produisent les différents biens en utilisant le travail et le capital considéré comme un bien importable. $\mathrm{Q}$ est la productivité total des facteurs et $\alpha i$ (avec $\mathrm{i}=\mathrm{x}, \mathrm{f}$, n) est le coefficient du travail dans les différents secteurs d'activité. $\left(K_{t}^{x}\right)$ et $\left(K_{t}^{f}\right)$ représentent le capital dans le secteur des exportations et des importations, respectivement, et $\left(K^{n}\right)$ représente le stock de capital fixe dans le secteur non échangeable. Les niveaux d'emploi dans les différents secteurs sont $\left(L^{x}\right)$, $\left(L^{f}\right)$ et $\left(L_{t}^{n}\right)$. Comme le capital, l'emploi est fixe dans le secteur échangeable. $(\phi)$ et ( $\delta$ ) sont le coefficient d'ajustement du capital et son taux de dépréciation, respectivement. Les avoirs extérieurs nets et le taux d'intérêt étranger sont notés $\left(A_{t}\right)$ et $\left(r^{*}\right)$. Le temps total disponible des ménages est $(T)$. $P^{x}$ est le prix moyen international, il s'agit du prix des importations pris ici comme numéraire.

Deux types de chocs sont à l'origine du cycle économique : des chocs de productivité sectorielle, notés $\left(e_{i}^{t}\right)$ avec $i=x, f$ et $n$, et des chocs sur les termes de l'échange $\left(e_{t}^{p}\right)$. La persistance et la volatilité des chocs sont définies de la manière suivante : $\rho_{e^{i}}=\rho_{e^{p}}=\theta, \sigma_{e^{i}}=e^{i}$, et $\sigma_{e^{p}}=e^{p}$. Les chocs se transmettent au niveau international à travers la mobilité internationale des capitaux, le coût des importations et le pouvoir d'achat des biens exportables.

Ces mécanismes de transmission internationale des fluctuations proviennent des chocs domestiques affectant la productivité des facteurs de production et des chocs sur les termes de l'échange. Pour ce dernier type de choc, le choix de modéliser des petites économies ouvertes est fondamental car cela permet de considérer les termes de l'échange comme une variable exogène et stochastique. Si des modèles de grandes économies ouvertes présentent l'avantage de rendre endogènes les 
termes de l'échange par l'équilibre de l'ensemble des marchés, ils ont l'inconvénient d'en sous-estimer très fortement la volatilité au cours du cycle. Mendoza (1995) peut au contraire exactement imposer à l'économie étudiée la volatilité observée des termes de l'échange et mesurer ainsi la contribution de ce choc exogène au cycle économique. Cette volatilité des termes de l'échange va constituer pour Mendoza (1995) la différence essentielle entre les pays industrialisés et les pays en développement.

Mendoza (1995) construit des séries annuelles de termes de l'échange, définies comme le rapport des valeurs unitaires des exportations sur celui des importations. La composante cyclique de ces séries, extraite par l'application du filtre HodrickPrescott, se révèle également persistante pour l'ensemble des économies considérées mais d'une volatilité très variable (cf. tableau 1 ci-dessous).

Tableau 1 : Propriétés macroéconomiques du cycle

\begin{tabular}{lccc|c}
\hline & \multicolumn{3}{c|}{ Données } & \multicolumn{2}{c}{ Modèle } \\
Pays développés (G7) & $\sigma(t o t)$ & $\sigma(y)$ & $\sigma(y) / \sigma(t o t)$ & 1.69 \\
Pays en développement & 5.37 & 9.14 & 1.30 & 0.86 \\
Pays africains & 12.44 & 14,00 & 1.00 & 0.47 \\
\hline
\end{tabular}

Sources : Mendoza (1995), extraits des tableaux 3, 7 et 8, pages 111, 123 et 124.

Note : tot $=$ termes de l'échange, $y=$ production, $\sigma(\mathrm{x})=$ écart-type de $\mathrm{x}$.

Pour le groupe des pays développés (ceux du G7), la moyenne de l'écart-type des termes de l'échange se situe à $5.37 \%$ en incluant le cas atypique du Japon dont l'écart-type est très supérieur à celui des autres économies. Pour l'ensemble des pays en développement, la moyenne de l'écart-type est très supérieure : $12.44 \%$ pour tous les pays développés et même $14.37 \%$ si l'on considère uniquement les pays africains. Les valeurs les plus importantes concernent les pays exportateurs de pétrole (Algérie, Nigéria, Arabie-Saoudite et Venezuela). Mendoza (1995) montre également que la volatilité relative des agrégats macroéconomiques par rapport à celle des termes de l'échange varie très peu entre les pays. L'écart-type moyen de la composante cyclique de la production est bien nettement supérieur dans les pays en développement que dans les pays industrialisés (14.00\% contre $9.14 \%$, respectivement), toutefois, si on le rapporte à l'écart type moyen de la composante 
cyclique des termes de l'échange les différences s'estompent fortement (1.30 contre 1.69, respectivement).

Pour quantifier les effets des chocs sur les termes de l'échange, Mendoza (1995) adapte l'étalonnage de son modèle aux situations moyennes observées dans les pays industrialisés, d'une part, et dans les pays en développement, d'autre part (cf. tableau 2 ci-dessous). Ces derniers sont caractérisés par des coefficients plus faibles du travail dans les fonctions de production, un degré d'aversion au risque plus important ${ }^{11}$, une plus grande élasticité de substitution entre les biens échangeables et non-échangeables et une dépendance négative entre les chocs sur les termes de l'échange et la productivité domestique. Ces différences d'étalonnage ont tendance à diminuer l'amplitude des fonctions de réponse des variables endogènes aux chocs par rapport à l'étalonnage retenu pour les pays industrialisés sans en changer pour autant la direction (excepté pour la consommation mesurée dans les prix des importations).

Au final, selon Mendoza (1995), les pays industrialisés et en développement ne se distinguent pas par des mécanismes de propagation substantiellement différents mais par l'amplitude des impulsions à l'origine des fluctuations. Les chocs sur les termes de l'échange, qui expliquent près de la moitié de la volatilité de la production en moyenne, sont nettement plus volatiles dans les pays en développement et à l'origine de la plus forte volatilité observée dans ces économies de la production, la consommation et de la balance commerciale.

\footnotetext{
${ }^{11}$ Les paramètres décrivant les préférences sont basés sur les estimations d'Ostry et Reinhart
} (1992). 
Tableau 2 : Différences d'étalonnage entre les pays développées (PD) et en développement (PVD)

\begin{tabular}{|c|c|c|c|}
\hline Paramètres & Symbole & PD & PVD \\
\hline $\begin{array}{l}\text { Ecart-type des chocs de productivité dans le secteur des } \\
\text { exportations }\end{array}$ & $e^{x}$ & 1.9 & 4.0 \\
\hline $\begin{array}{l}\text { Ecart-type des chocs de productivité dans le secteur des } \\
\text { importations }\end{array}$ & $e^{f}$ & 1.9 & 4.0 \\
\hline $\begin{array}{l}\text { Ecart-type des chocs de productivité dans le secteur non- } \\
\text { échangeable }\end{array}$ & $e^{n}$ & 1.4 & 3.8 \\
\hline Chocs sur les termes de l'échange & $e^{p}$ & 4.7 & 11.77 \\
\hline Persistance des chocs & $\theta$ & 0.473 & 0.414 \\
\hline Corrélation entre les chocs & $\rho_{e^{x}, e^{p}}$ & 0.165 & -0.46 \\
\hline Taux d'intérêt extérieur & $r^{*}$ & 0.04 & 0.04 \\
\hline Coefficient du travail dans le secteur des exportations & $\alpha x$ & 0.51 & 0.429 \\
\hline Coefficient du travail dans le secteur des importations & $\alpha f$ & 0.73 & 0.302 \\
\hline Coefficient du travail dans le secteur non échangeable & $\alpha n$ & 0.56 & 0.34 \\
\hline Coefficient de dépréciation du capital & $\delta$ & 0.1 & 0.1 \\
\hline Coefficient d'ajustement du capital & $\phi$ & 0.028 & 0.028 \\
\hline Productivité totale des facteurs & $Q$ & 1.0 & 0.3 \\
\hline Elasticité de substitution de la consommation agrégée & $1 / \gamma$ & 0.66 & 0.38 \\
\hline Elasticité de substitution entre les biens échangeables & $1 /(\mu+1)$ & 0.74 & -0.82 \\
\hline Part des exportations dans les dépenses échangeables & $a$ & 0.3 & 0.15 \\
\hline Coefficient d'élasticité de l'offre du travail & $\omega$ & 2.08 & 0.786 \\
\hline Coefficient de préférence inter-temporelle & $\beta$ & 0.009 & 0.009 \\
\hline
\end{tabular}

Source : Mendoza (1995), pages 119-122.

Note : La structure des chocs est calculée à partir des données de l'échantillon, alors que les paramètres des préférences et des technologies de production sont repris de Kravis et al. (1982) et Stockman et al. (1990) pour les pays développés et Ostry et al. (1992) pour les pays en développement. 


\subsection{Les chocs sur les prix relatifs mondiaux et le taux d'intérêt [Kose et Reizman (2001)]}

Kose et Reizman (2001) poursuivent la voie ouverte par Mendoza (1995), mettant en avant le rôle des facteurs internationaux dans le cycle des pays d'ASS, en se distinguant principalement sur la définition des chocs. Au lieu des chocs sur les termes de l'échange considérés par Mendoza (1995), Kose et Reizman (2001) introduisent des chocs sur deux prix relatifs mondiaux afin de mieux refléter l'environnement économique en ASS.

Les deux prix relatifs mondiaux sont définis par le rapport des prix des biens intensifs en capital sur celui des biens primaires et celui des prix des biens intermédiaires importés également par rapport aux prix des biens primaires. Kose et Reizman (2001) justifient cette approche par le fait que les économies africaines sont très dépendantes du commerce extérieur, avec des exportations constituées essentiellement des biens primaires, très fluctuantes à cause de la volatilité de leurs prix, et des importations constituées principalement des biens intensifs en capital et des biens intermédiaires. Une propriété importante des prix relatifs par rapport aux termes de l'échange est leur plus forte volatilité et leur plus grande persistance.

Pour évaluer la contribution de ces différents chocs au cycle économique, Kose et Reizman (2001) développent un modèle de petite économie ouverte similaire à celui de Mendoza (1995) à l'exception des contraintes sur la répartition du capital physique entre les différents secteurs d'activité. En plus des chocs sur les prix relatifs, Kose et Reizman (2001) introduisent des chocs sur la productivité des facteurs et un choc sur le taux d'intérêt mondial, facteur ignoré par Mendoza (1995). La prise en compte des chocs sur le taux d'intérêt s'explique par le fait qu'en recourant à l'endettement extérieur pour financer leurs importations, les économies africaines doivent faire face au service de la dette et s'exposent de ce fait aux variations du taux d'intérêt mondial.

Le modèle décrit une économie produisant des biens de consommation finale non échangeables et des biens primaires. La production des biens de consommation finale $y_{t}^{f}$ se fait en utilisant le capital $k_{t}^{f}$, le travail $n_{t}^{f}$ et des biens intermédiaires de production $v_{t}$. L'équation suivante décrit la technologie de production utilisée : 
$y_{t}^{f}=z_{t}^{f}\left(n_{t}^{f}\right)^{\alpha}\left[s\left(k_{t}^{f}\right)^{-u}+(1-s) v_{t}^{-u}\right]^{-\frac{1-\alpha}{u}} \quad v<\alpha, s, u<1$

où $z_{t}^{f}$ représente les chocs exogènes sur la productivité, $\alpha$ le coefficient d'élasticité du travail dans la production non échangeable, $s$ le poids relatif du capital par rapport aux biens intermédiaires et $u$ l'élasticité de substitution entre les biens intermédiaires de production et le capital. Les firmes du secteur primaire produisent $y_{t}^{p}$ en utilisant le travail $n_{t}^{p}$, le capital $k_{t}^{p}$ et la terre $L^{p}$ dont l'offre est supposée inélastique. La fonction de production des biens primaires est décrite par l'équation suivante :

$y_{t}^{p}=z_{t}^{p}\left(n_{t}^{p}\right)^{\theta_{1}}\left(k_{t}^{p}\right)^{\theta_{2}}\left(L^{p}\right)^{1-\theta_{1}-\theta_{2}} \quad 0<\theta_{1}, \theta_{2}<1$

où $z_{t}^{p}$ est le choc technologique, $\theta_{1}$ et $\theta_{2}$ sont respectivement les coefficients d'élasticité du travail et du capital dans la production échangeable. L'accumulation du capital dans les deux secteurs est décrite de façon suivante :

$k_{t+1}^{j}=(1-\delta) k_{t}^{j}+\emptyset_{j}\left(\frac{i_{t}^{j}}{k_{t}^{j}}\right) k_{t}^{j} j=f, p$

où $\delta$ est le taux de dépréciation, $i_{t}^{j}$ le niveau d'investissement dans le secteur $\mathrm{j}$ et $\emptyset_{j}($.) la fonction concave du coût d'ajustement du capital. La contrainte budgétaire dans le secteur non échangeable est donnée par la relation suivante :

$c_{t}+i_{t}^{f}=y_{t}^{f}$

Le modèle est étalonné et le processus générateur des chocs estimé à partir des séries de prix relatifs et de productivité afin de reproduire au mieux le comportement moyen d'un échantillon de vingt-deux pays d'ASS ${ }^{12}$. Les chocs sur les prix relatifs affectent directement les recettes d'exportation et se transmettent aux agrégats macroéconomiques (production, consommation et investissement) via les importations de biens intermédiaires (intensifs en capital). Le modèle reproduit assez bien la quasi-totalité des composantes du cycle étudiées, aussi bien au niveau agrégé que sectoriel, à l'exception de la consommation. Pour comprendre la forte volatilité des économies africaines, Kose et Reizman (2001) mesurent la contribution respective de chaque choc au cycle économique.

12 Kose (2002) complète les résultats de Kose et Reizman (2001) pour un ensemble plus large de pays en développement. 
Ceci conduit Kose et Reizman (2001) aux mêmes conclusions que Mendoza (1995) : les chocs internationaux sur les prix expliquent près de la moitié de la volatilité de la production (cf tableau 3 ci-dessous). La structure particulière du commerce internationale des économies africaines les exposent plus aux chocs mondiaux que les autres économies et expliquent ainsi leur plus grande volatilité. L'intérêt de la contribution de Kose et Reizman (2001) est d'apporter cette explication dans un cadre théorique et empirique plus conforme à la structure des économies africaines. Cette contribution apporte également un second résultat important: la faible contribution des chocs de taux d'intérêt à la variance de la production (moins de $1 \%)^{13}$. La finance internationale, en tant que source d'impulsion, ne paraît pas jouer un rôle déterminant dans le cycle économique. Par contre, comme nous le verrons avec Özbilgin (2010), les contraintes d'accès à la finance internationale peuvent constituer un puissant mécanisme de propagation dans ces économies.

Tableau 3 : Décomposition de la variance

\begin{tabular}{lcc}
\hline Variable & Chocs commerciaux & chocs technologiques \\
\hline Production & 44.64 & 54.49 \\
Consommation & 79.14 & 17.97 \\
Investissement & 86.36 & 13.17 \\
Balance commerciale & 73.72 & 21.71
\end{tabular}

Source : Kose et al. (2001) extrait du tableau 6 page 71.

\subsection{Les chocs sur l'aide au développement [Arellano et al. (2009)]}

Arellano et al. (2009) mettent également en avant les facteurs externes pour expliquer le comportement du cycle économique en Afrique, mais contrairement à Mendoza (1995) et Kose et Reizman (2001), ces auteurs ne considèrent pas le rôle des prix des biens échangés, mais celui de l'aide au développement. L'efficacité de l'aide au développement est un sujet majeur en économie du développement qui a été très vivement débattu sur les plans théoriques, empiriques et de politique économique (voir notamment Word Bank (1998), Estearly (2003) et Rajan et Subramanian (2008)). La volatilité de l'aide et sa faible prédictibilité ont été

13 Le reste de la variance de la production s'explique par les chocs de productivité. 
identifiées comme une source d'inefficacité du système d'aide internationale à destination des pays en développement ; voir Bulir et Hamann (2007) pour une étude empirique récente. La contribution d'Arellano et al. (2009) à ces débats est d'évaluer les conséquences de la volatilité de l'aide dans un modèle DSGE.

Le modèle développé par les auteurs est celui d'une petite économie ouverte. L'aide est modélisée comme un transfert stochastique de biens échangeables qui intervient comme une ressource dans la contrainte budgétaire des ménages. Les investissements sont considérés comme des biens échangeables, de telle sorte que l'aide peut être utilisée directement pour des investissements. Le pays n'ayant pas accès aux actifs extérieurs, la seule façon d'épargner est d'investir dans le stock de capital domestique. Par l'existence de deux secteurs (produisant un bien échangeable $(T)$ et un bien non-échangeable $(N)$ ), l'aide internationale crée un phénomène dit de "Dutch disease" orientant les facteurs de production domestiques vers le secteur non échangeable. La contrainte budgétaire exprimée en termes des biens échangeables est alors définie par l'équation suivante :

$C_{t}^{T}+p_{t}^{N} C_{t}^{N}=r_{t} K_{t}+w_{t} L_{t}-i_{t}+X_{t}$

Où $C_{t}^{T}, p_{t}^{N}, C_{t}^{N}, r_{t}, K_{t}, w_{t}, L_{t}, i_{t}, X_{t}$ sont respectivement la consommation des biens échangeables, le prix relatif des biens non échangeables exprimé en termes des biens échangeables, la consommation des biens non échangeables, le taux d'intérêt réel domestique payé sur le capital, le stock du capital dans l'économie, le taux de salaire réel, le taux d'emploi, l'investissement et les afflux d'aides stochastiques à l'économie. Les conditions d'équilibre des marchés sont :

$C_{t}^{N}=Y_{t}^{N}$

$C_{t}^{T}+i_{t}=Y_{t}^{T}+X_{t}$

L'aide est une ressource uniquement sur le marché des biens échangeables (13).

Arellano et al. (2009) appliquent leur modèle à la Côte d'Ivoire et étalonnent les paramètres structurels afin de reproduire plusieurs caractéristiques de cette économie sur la période 1991-2005. Le modèle reproduit correctement les écarttypes et les corrélations de la production totale, l'aide, la consommation et l'investissement (cf. tableau 4 ci-dessous). Les auteurs utilisent ce modèle pour montrer les effets sur le cycle d'une augmentation du poids de l'aide dans l'économie. Recevoir une aide en moyenne plus importante augmente la volatilité de 
la consommation, de l'investissement et du taux de change réel et renforce la corrélation de l'ensemble des variables macroéconomiques avec l'aide reçue.

Tableau 4 : Ecart-type des composantes du cycle en Côte d'Ivoire

\begin{tabular}{lccccccc}
\hline Variables & données & modèle & TFP (chocs) & Aide (chocs) & \multicolumn{3}{c}{ Sensibilité à l'aide } \\
\hline & & & & & $0 \%$ & $10 \%$ & $20 \%$ \\
PIB & 0.11 & 0.11 & 0.10 & 0.02 & 0.11 & 0.11 & 0.13 \\
Aide & 0.72 & 0.73 & 0.00 & 0.73 & N/A & 0.73 & 0.73 \\
Prix relatifs (N) & 0.12 & 0.04 & 0.03 & 0.04 & 0.03 & 0.05 & 0.12 \\
PIB échangeable & 0.10 & 0.15 & 0.14 & 0.04 & 0.14 & 0.15 & 0.23 \\
PIB non échangeable & 0.06 & 0.11 & 0.11 & 0.03 & 0.11 & 0.12 & 0.13 \\
Consommation & 0.11 & 0.11 & 0.10 & 0.04 & 0.11 & 0.11 & 0.14 \\
Investissement & 0.23 & 0.32 & 0.26 & 0.05 & 0.31 & 0.37 & 0.54
\end{tabular}

Source : Arellano et al. (2009), extraits des tableaux 3, 4 et 5 pages 93, 94 et 95.

Contrairement à Mendoza (1995) et Kose et Reizman (2002), les chocs de productivité expliquent la quasi-totalité du cycle économique chez Arellano et al. (2009). Annuler la volatilité de l'aide aurait un impact marginal sur les fluctuations dans l'économie ${ }^{14}$. En termes normatifs, les auteurs reportent de très forts gains en bien-être (de l'ordre de 3.3\% de la consommation d'état stationnaire) par le maintien d'une aide volatile mais très fortement contra-cyclique qui permettrait d'assurer les ménages contre les chocs de productivité.

\subsection{Les conséquences de la participation limitée aux marchés financiers internationaux [Özbilgin (2010)]}

Tandis que les trois précédentes contributions mettent l'accent sur des sources d'impulsion spécifiques aux pays d'ASS, Özbilgin (2010) explore le rôle de la participation limitée aux marchés financiers comme mécanisme de propagation des chocs technologiques spécifique à ces pays.

Özbilgin (2010) fonde son modèle sur des observations empiriques montrant le faible développement des marchés financiers dans les pays en développement ainsi que la plus forte prévalence de ménages ne détenant pas d'actifs financiers et

14 L'écart-type de la production de la Côte d'Ivoire est de 0.11 dans le modèle avec les deux chocs. L'écart type est de 0.1092 en présence des seuls chocs technologiques et de 0.02 en présence des seuls chocs affectant l'aide au développement, cf. tableau 4. 
consommant l'intégralité de leur revenu dans ces pays. Pour prendre en compte ce phénomène, Özbilgin (2010) introduit dans le modèle traditionnel de petite économie ouverte des agents qui se différencient en termes d'accès aux marchés financiers. Les agents de type A participent pleinement aux marchés financiers, ils sont des détenteurs du capital physique et peuvent également acheter ou vendre des actifs financiers sur le marché international. Les agents de type B n'ont pas accès au marché du capital physique mais peuvent toutefois substituer leurs consommations intertemporelles sur le marché international des actifs financiers. Les agents de type $\mathrm{C}$ ne participent à aucun marché financier et consomment intégralement leur revenu à chaque période. Sous ces hypothèses, le programme de maximisation d'utilité du ménage de type-i s'écrit :

$\operatorname{Max} E_{0} \sum_{t=0}^{\infty} \beta^{t} U_{i}\left(c_{i, t} n_{i, t}\right) \quad i=A, B, C$

sous la contrainte

$c_{i, t}+\left[k_{t+1}-(1-\delta) k_{t}+\phi\left(k_{t+1}-k_{t}\right)\right] I_{\{A\}}(i)+b_{i, t}\left(1+r_{i, t}\right) I_{\{A, B\}}(i) \leq w_{t} n_{i, t}+$ $q_{t} k_{t} I_{\{A\}}(i)+b_{i, t+1} I_{\{A, B\}}(i)$

Les équations (14) et (15) représentent respectivement les fonctions d'utilité et la contrainte budgétaire du ménage représentatif, où $c_{i, t}, n_{i, t}, b_{i, t}, k_{t}$ et $U_{i}$ sont respectivement la consommation, le travail, les titres, le capital et la fonction d'utilité. $I_{M}($.) est une variable indicatrice qui prend la valeur 0 ou 1 selon le type de ménage A,B ou C.

Özbilgin (2010) compare ensuite les prédictions du modèle par rapport à la situation moyenne observée dans les pays en développement selon la présence ou non d'une participation limitée des agents. Le cas standard d'absence de participation limitée correspond à la situation où l'économie est entièrement peuplée d'agents de type $A$. La participation limitée améliore les performances du modèle en augmentant la volatilité prédite de la consommation et la contra-cyclicité de la balance commerciale (cf. tableau 5 ci-dessous). Au final, la différence entre les petites économies développées et en développement ne résulte pas tant d'une différence d'exposition à des chocs, mais au fait que la participation limitée aux marchés financiers dans celles en développement modifie la réaction de certaines variables (comme la consommation et la balance commerciale) à ces chocs. Par contre, la forte volatilité des économies ne s'explique pas ici par cette participation limitée. Dans le modèle d'Özbilgin (2010), augmenter la participation aux marchés 
financiers accroît en effet la volatilité de la production dans l'économie (cf. la dernière colonne du tableau 5).

Tableau 5 : Propriétés du cycle économique selon la participation aux marchés financiers

\begin{tabular}{clcccc}
\hline Symbole & Variables & Données & $\begin{array}{c}\text { Modèle } \\
\text { standard }\end{array}$ & $\begin{array}{c}\text { Participation limitée } \\
\lambda_{A}=0.03 \lambda_{B}=0.17\end{array}$ & $\begin{array}{c}\text { Participation limitée } \\
\lambda_{A}=0.25 \lambda_{B}=0.5\end{array}$ \\
\hline$\sigma_{y}$ & Volatilité de la production & 4.07 & 4.07 & 4.07 & 4.25 \\
$\sigma_{c} / \sigma_{y}$ & Volatilité de la consommation & 1.28 & 0.75 & 0.85 & 0.72 \\
$\sigma_{i} / \sigma_{y}$ & Volatilité de l'investissement & 4.18 & 4.18 & 4.18 & 6.10 \\
$\sigma_{t b} / \sigma_{y}$ & Volatilité de la balance com & 0.87 & 0.81 & 0.81 & 1.29 \\
$\rho_{c, y}$ & Corr production-consommation & 0.69 & 0.88 & 0.98 & 0.94 \\
$\rho_{i, y}$ & Corr production-investissement & 0.60 & 0.57 & 0.55 & 0.39 \\
$\rho_{t b, y}$ & Corr production-balance com & -0.20 & -0.04 & -0.20 & -0.04 \\
$\sigma_{c A} / \sigma_{Y}$ & Volatilité de conso de type-A & - & - & 1.06 & - \\
$\sigma_{c B} / \sigma_{y}$ & Volatilité de conso de type-B & - & - & 0.69 & - \\
$\sigma_{c A} / \sigma_{y}$ & Volatilité de conso de type-C & - & - & 1.00 & - \\
\hline
\end{tabular}

Source : Özbilgin (2010), extraits des tableaux 3 et 6 pages 134 et 136.

Note : dans le modèle à participation limitée, les valeurs de $\lambda_{A}$ et $\lambda_{B}$ sont respectivement de 0.03 et 0.07 .

\section{Les politiques conjoncturelles en ASS}

Les travaux présentés dans la section précédente montrent l'intérêt des modèles DSGE pour expliquer les spécificités du cycle économique en ASS. Dans cette section, nous montrons comment ces modèles peuvent être utilisés pour l'évaluation des politiques conjoncturelles.

\subsection{Une estimation de la règle de politique monétaire au Ghana [Houssa et al. (2010)]}

Houssa et al. (2010) apportent une estimation de la règle de Taylor régissant la politique monétaire pour un pays d'ASS, le Ghana. Par rapport aux études présentées dans la section précédente, la contribution de Houssa et al. (2010) se distingue par la modélisation de la sphère nominale et le recours aux méthodes d'estimation bayésienne ${ }^{15}$.

\footnotetext{
${ }^{15}$ Voir An et Schorfeide (2007) pour une présentation de ces méthodes.
} 
Houssa et al. (2010) s'inscrivent dans la lignée des modèles DSGE de taille moyenne ("medium-scale ") avec des frictions réelles et nominales développés par Smets et Wouters (2007) et Christiano et al. (2005) pour l'économie américaine. Plus précisément, ils reprennent la version de ces modèles proposée par Adolfson et al. (2008) pour des petites économies ouvertes ${ }^{16}$. Les marchés des biens et du travail sont en concurrence monopolistique et les prix des biens caractérisés par une rigidité nominale. La spécificité des économies africaines pour les auteurs réside plus dans les chocs frappant ces économies que dans les mécanismes de propagation et d'amplification. Cela les amène à considérer un grand nombre de chocs (onze) dont ils estiment les lois d'évolution à partir des méthodes d'estimation bayésienne sur la période 1983(3)-1997(4). La politique monétaire est approximée suivant la règle cidessous exprimée sous forme log-linéaire :

$\hat{R}_{t}=\rho_{R} \hat{R}_{t-1}+\left(1-\rho_{R}\right)\left(\hat{\bar{\pi}}_{t}+r_{\pi}\left(\hat{\pi}_{t-1}-\hat{\bar{\pi}}_{t}\right)+r_{y} \hat{y}_{t-1}+r_{x} \hat{x}_{t-1}+r_{m} \hat{m}_{t-1}\right)+\epsilon_{r, t}$

où $\hat{R}_{t}$ est le taux d'intérêt de court terme et $\hat{\pi}_{t}$ est l'indice des prix de consommation. $\hat{y}_{t}, \hat{x}_{t}$, et $\widehat{m}_{t}$ sont respectivement les déviations de la production, des exportations et des importations par rapport à l'état stationnaire. $\widehat{\bar{\pi}}_{t}$ est l'objectif d'inflation et $\epsilon_{r, t}$ les chocs sur le taux d'intérêt. La déviation de la cible d'inflation est donnée par la relation suivante :

$\hat{\bar{\pi}}_{t}^{c}=\rho_{\pi} \hat{\bar{\pi}}_{t-1}^{c}+\epsilon_{\hat{\bar{\pi}}^{c}, t}^{c}$

Les résultats de leurs estimations indiquent que les chocs technologiques permanents expliquent la quasi-totalité de la variance des importations (98\%) et des exportations (97\%) ainsi qu'une part très importante de celles de l'inflation (83\%), du taux d'intérêt (52\%) et de la production (50\%) (cf. tableau 6 ci-dessous). Les chocs de dépenses publiques transitoires expliquent la quasi-totalité du reste de la variance de la production et du taux d'intérêt. Les chocs monétaires jouent un rôle essentiellement pour l'inflation. Ces résultats donnent un rôle prépondérant aux facteurs domestiques (productivité et dépenses publiques) qui contrastent avec les conclusions des travaux présentés dans la section précédente de cette revue. Leur estimation porte également sur les paramètres de la règle de Taylor. La règle de

\footnotetext{
${ }^{16}$ Adolfson et al. (2008) étudient la Suède.
} 
Taylor décrite par l'équation (16) estimée ${ }^{17}$ donne un poids très important à la stabilisation de la production et des coefficients de réaction quasi-nuls du taux d'intérêt à l'inflation, aux exportations et aux importations.

Tableau 6 : Décomposition de la variance

\begin{tabular}{lccccc}
\hline & \multicolumn{5}{c}{ Variables endogènes } \\
\cline { 2 - 5 } Chocs & Inflation & Production & Intérêt & Importations & Exportations \\
\hline Investissement & 0 & 0.01 & 0 & 0.01 & 0 \\
Technologique transitoire & 0 & 0 & 0 & 0 & 0 \\
Technologique permanent & 83.33 & 50.87 & 52.12 & 98.29 & 97.41 \\
Marge domestique & 0.02 & 0.06 & 0.06 & 0.24 & 0.03 \\
Marge (importation) & 0.01 & 0 & 0 & 0.04 & 0.01 \\
Marge (exportation) & 0.02 & 0.06 & 0.06 & 0.25 & 0.11 \\
Taux d'intérêt étranger & 1.97 & 0.05 & 0.05 & 1.1 & 2.3 \\
Choc monétaire & 14.57 & 0.33 & 0.25 & 0 & 0.04 \\
Taxe sur le revenu & 0 & 0 & 0 & 0 & 0 \\
Taxe sur la consommation & 0 & 0 & 0 & 0 & 0 \\
Dépenses du Gouvernement & 0.06 & 48.33 & 47.42 & 0.07 & 0.07 \\
\hline Source : Houssa et al. (2010), tableaury
\end{tabular}

Source : Houssa et al. (2010), tableau 1 page 143.

L'exercice d'Houssa et al. (2010) leur permet d'identifier les spécificités des chocs frappant l'économie du Ghana et d'estimer la politique monétaire suivie dans ce pays. Les auteurs soulignent également les limites de leur approche. Même si pour ce pays ils disposent d'un échantillon de séries trimestrielles conséquent (14 années) de nombreux paramètres sont insuffisamment identifiés par la procédure d'estimation. De plus, un traitement plus fin des finances publiques dans ce modèle avec notamment une meilleure prise en compte de la fiscalité, serait nécessaire pour comprendre l'origine du poids des chocs sur les dépenses publiques.

17 Cette estimation donne un coefficient d'élasticité de 6 pour la production, alors qu'il est de 0 pour les exportations. Le coefficient estimé d'élasticité de l'inflation est quant à lui inférieur à 1, afin de ne pas violer le principe de Taylor selon lequel ce coefficient doit être supérieur à l'unité, les auteurs le fixe à 1.5 . 


\subsection{Une estimation de la règle de politique monétaire au Mozambique [Peiris et Saxegaard (2010)]}

Peiris et Saxegaard (2010) poursuivent le même objectif que Houssa et al. (2010) : étudier la politique monétaire dans un modèle DSGE réel et monétaire estimé pour une économie d'ASS. Les auteurs s'inscrivent explicitement dans l'esprit de la « science de la politique monétaire » développée par Clarida et al. (1999) qu'ils souhaitent étendre aux pays d'ASS en intégrant les spécificités de ces économies à savoir : (i) le rôle du secteur échangeable et le phénomène de "dutch disease » et (ii) le rôle prépondérant de la base monétaire par rapport au taux d'intérêt compte tenu du faible développement du marché monétaire interbancaire et des marchés secondaires de la dette publique.

Dans le modèle proposé par Peiris et Saxegaard (2010), l'équation suivante spécifie les règles de politiques monétaires menées par la banque centrale dans ses interventions sur le marché de change :

$\Delta Z_{t}=z_{1}\left(Z-Z_{t-1}\right)+\left(1-z_{2} \omega\right)\left(A_{t}-A\right)+z_{3} \log \left(\frac{e_{t} / e_{t-1}}{\pi / \pi^{*}}\right)+z_{4} \log \left(\frac{\pi_{t}}{\pi}\right)+u_{t}^{z}$

où $\Delta Z_{t}$ est la variation des devises. $Z_{t}$ et $A_{t}$ sont respectivement le niveau des réserves et de l'aide internationale à période $\mathrm{t}(Z$ et $A$ représentent les valeurs d'état stationnaire). La part de l'aide dévolue à la réduction des taxes est $\omega . z_{1}$ représente l'engagement des autorités à maintenir un niveau constant de réserve en devises. $z_{2}$ est le poids des interventions sur le marché de change en fonction des hausses des dépenses gouvernementales financées par les afflux d'aide. La prise en compte des différentielles d'inflation (nationale et extérieure) est pondérée par $z_{3}$ et celle de la deviation de l'inflation par rapport au régime permanent par $z_{4}$. $u_{t}^{z}$ est le choc sur les réserves extérieures.

Peiris et Saxegaard (2010) considèrent alternativement trois règles de politique monétaire. La première cherche à stabiliser le taux de change $\left(z_{3}\right)$, la seconde le taux de croissance des prix à la consommation $\left(z_{4}\right)$ et la troisième le taux de croissance des prix du secteur non-échangeables $\left(z_{2}\right)$. Le modèle comprend des rigidités réelles et nominales habituelles pour cette classe de modèle (prix visqueux, concurrence monopolistique, coût d'ajustement du capital), un secteur bancaire explicite et des frictions financières qui pèsent sur l'investissement des entreprises. 
Le modèle est ensuite estimé par les méthodes d'économétrie bayésienne pour le Mozambique sur la période 1996(1)-2005(4). Les auteurs utilisent dix-huit séries macroéconomiques et introduisent quatorze chocs dans le modèle. Ils comparent ensuite la volatilité des principaux agrégats macroéconomiques pour les trois règles de politique monétaire précédemment définies compte tenu des valeurs des paramètres structurels estimées. II ressort de cet exercice (cf. tableau 7 ci-dessous) que la politique de ciblage de l'inflation des prix à la consommation est celle qui stabilise le plus la production, la consommation et l'inflation. Elle accroît par contre la volatilité des exportations nettes, du taux de change et du taux d'intérêt. En termes de bien-être, compte tenu du coût pour l'agent représentatif des fluctuations de sa consommation, cette politique se révèle être la meilleure.

Tableau7 : Volatilité des variables macroéconomiques sous différents objectifs de politique monétaire

\begin{tabular}{ccccccccc}
\hline & PIB & Consommation & $\begin{array}{c}\text { Exportations } \\
\text { nettes }\end{array}$ & $\begin{array}{c}\text { Inflation } \\
\text { (IPC) }\end{array}$ & $\begin{array}{c}\text { Taux de } \\
\text { change } \\
\text { nominal }\end{array}$ & $\begin{array}{c}\text { Taux de } \\
\text { change } \\
\text { réel }\end{array}$ & $\begin{array}{c}\text { Taux } \\
\text { d'intérêt }\end{array}$ & $\begin{array}{c}\text { Bien } \\
\text { être }\end{array}$ \\
\hline$z_{4}$ & 0.4822 & 0.3023 & 0.0438 & 0.0049 & 0.0424 & 0.0518 & 0.0369 & -7.2561 \\
$z_{2}$ & 0.4842 & 0.3025 & 0.0425 & 0.0103 & 0.0382 & 0.0510 & 0.0355 & -7.2578 \\
$z_{3}$ & 0.4892 & 0.3033 & 0.0541 & 0.0391 & 0.0072 & 0.0521 & 0.0410 & -7.2861 \\
\hline
\end{tabular}

Source : Peiris et Saxegaard (2010), tableau 1 page 19.

Note $: z_{3}=$ objectif de taux de change $; z_{2}=$ objectif d'inflation secteur (N) $; z_{4}=$ objectif d'inflation (IPC).

\subsection{La politique monétaire et fiscale face aux afflux d'aide [Adam et al. (2009)]}

Adam et al. (2009) traitent également de la politique monétaire, comme les deux études précédemment décrites, mais se concentrent sur la question de l'aide au développement. Ils étudient plusieurs règles de politique économique pour faire face aux afflux d'aide internationale dans le contexte d'une intégration limitée dans le système financier international et d'une faible convertibilité des monnaies. Dans leur approche, l'aide intervient comme une ressource budgétaire pour l'Etat et non pas comme une source de financement explicite de programmes de développement. 
Le modèle $\mathrm{DSGE}^{18}$ proposé par Adam et al. (2009) est celui d'une petite économie ouverte où le ménage représentatif consomme des biens importés et des biens non échangeables et répartit son épargne entre plusieurs actifs : la monnaie nationale, les devises étrangères et les titres gouvernementaux. II n'existe pas de banque dans l'économie de telle sorte que la monnaie est uniquement constituée de la base monétaire. Lorsque survient des chocs sur l'aide au développement, les autorités publiques réajustent leur contrainte budgétaire consolidée en intervenant sur les différents marchés de titres, nationaux et internationaux, et modifient donc la base monétaire et l'inflation dans l'économie. La politique fiscale est décrite par un premier paramètre qui indique la part de l'aide allouée à la dépense gouvernementale, le reste étant alloué à la réduction du déficit. Un second paramètre mesure le degré de lissage de la réduction du déficit opérée qui peut être instantanée ou étalée dans le temps. La politique de gestion des réserves est décrite par une règle d'accumulation des réserves qui dépend de la réaction des autorités aux variations du taux de change réel et de l'aide. Enfin, le déficit net de l'aide est intégré dans la règle d'émission de titres publics de l'État. Plus précisément, la règle d'intervention sur le marché de change est la suivante :

$\frac{\Delta z_{t}}{\bar{z}}=-\alpha_{1} \frac{x_{t}-\bar{x}}{\bar{x}}-\alpha_{2} \frac{z_{t-1}-\bar{z}}{\bar{z}}+\alpha_{3} \frac{\left(a_{t}-\bar{a}\right)-\gamma \cdot\left(d_{t}-\bar{d}\right)}{\bar{z}}$

où $x_{t}$ et $\bar{x}$ sont respectivement le taux de change à la période t et à l'état stationnaire. $z_{t}$ et $a_{t}$ sont respectivement les niveaux des réserves et de l'aide internationale (avec $\bar{z}$ et $\bar{a}$ à l'état stationnaire). Le déficit fiscal est $d_{t}-\bar{d}$. Les contraintes sur les paramètres régissant la politique monétaire sont : $\alpha_{1} \geq 0, \alpha_{2}>0, \alpha_{3} \in[0,1]$ et $0 \leq \gamma \leq$ 1.

Le modèle est ensuite utilisé pour simuler les différentes règles de politique monétaire définies ci-dessus, basée sur la variation des réserves détenues par les autorités. Les autorités peuvent ainsi laisser flotter la monnaie, c'est-à-dire qu'elles n'ont aucun soucis sur la stabilité du taux de change, dans ce cas les réserves disponibles ne changent pas. Les autorités peuvent aussi décider d'intervenir pour lutter contre la dépréciation de la monnaie en s'engageant à utiliser un montant

${ }^{18}$ Dans Buffie et al. (2008), les mêmes auteurs étudient les effets de l'aide au cours de la dynamique de transition et à long terme dans un modèle d'équilibre général dynamique et déterministe. 
déterminé des réserves disponibles. Enfin, les autorités peuvent adopter une politique mixte, qui consiste à laisser flotter la monnaie et d'intervenir seulement en cas de chocs sur l'aide, de telle sorte que l'aide non utilisée à la période courante est conservée comme réserve. Ces différentes politiques sont mises en œuvre sous l'hypothèse que les taxes ne changent pas et que les autorités déterminent le moment et le montant de l'aide dévolue à la réduction du déficit.

Le tableau 8 ci-dessous présente la volatilité des variables macroéconomiques induite par des chocs d'aide selon la politique économique suivie

Tableau 8 : Écart-type des variables macroéconomiques selon les politiques suivies

\begin{tabular}{c|cc|cc}
\hline & \multicolumn{2}{|c|}{ Aide entièrement dépensée } & \multicolumn{2}{c}{ Aide utilisée pour la réduction du déficit } \\
\hline & $\begin{array}{c}\text { Politique de } \\
\text { change mixte }\end{array}$ & $\begin{array}{c}\text { Politique de } \\
\text { cible de change }\end{array}$ & $\begin{array}{c}\text { Politique de } \\
\text { Change flexible }\end{array}$ & $\begin{array}{c}\text { Politique de } \\
\text { cible de change }\end{array}$ \\
\hline In & 2.491 & 1.324 & 11.260 & 1.596 \\
RER & 3.071 & 0.524 & 14.290 & 1.670 \\
RIR & 4.549 & 3.406 & 8.733 & 2.984 \\
Ca & 2.305 & 2.085 & 1.390 & 1.629 \\
DN & 0.787 & 0.914 & 0.821 & 1.154 \\
C & 0.874 & 1.109 & 1.937 & 0.713 \\
Dz & 3.077 & 2.949 & 2.286 & 2.256 \\
mg & 0.000 & 5.507 & 0.000 & 14.197 \\
\hline
\end{tabular}

Source : Adam et al. (2009), extraits des tableaux 3 à 6 pages 474-483.

Note : Inflation (In), taux de change nominal (NER), taux de change réel (RER), taux d'intérêt réel (RIR), surplus du compte courant $(\mathrm{Ca})$, production du secteur non échangeable (DN), consommation privée $(\mathrm{C})$ variation des devises (Dz), croissance de la masse monétaire nominale $(\mathrm{mg})$.

A la lumière de ces résultats, Adam et al. (2009) recommandent de dépenser complètement l'aide lorsqu'elle est reçue afin de limiter les réallocations d'actifs dans l'économie. Avec cette politique budgétaire, le choix de la politique de change n'est pas déterminant pour la stabilisation de l'économie. Si par contre l'aide est partiellement utilisée pour la réduction des déficits, s'écarter d'une politique de taux de change flottants pure stabilise l'économie. Par rapport aux politiques fiscales et de taux de change, les auteurs concluent que la politique de stérilisation des titres publics joue un rôle secondaire pour stabiliser l'économie face aux afflux d'aide. 


\subsection{La politique monétaire et fiscal face aux revenus pétroliers [Dagher et al.}

(2010)]

Comme Adam et al. (2009), Dagher et al. (2010) étudient les implications des variations de revenu sur l'efficacité des règles de politique monétaire et fiscale. Leurs approches se distinguent sur la nature du revenu. Chez Dagher et al. (2010), ces variations de revenu résultent de l'exploitation d'une ressource naturelle, le pétrole, et non de l'aide internationale. Si l'interprétation de la source de revenu est différente, sa modélisation est proche de celle de l'aide au développement. Dagher et al. (2010) proposent d'ailleurs une version stochastique du modèle déterministe de Berg et al. (2010) proposé initialement pour analyser les effets macroéconomiques des flux d'aide au développement.

Dagher et al. (2010) appliquent leur modèle au Ghana pour simuler les conséquences de la prochaine exploitation pétrolière sur cette économie. II était prévu que le Ghana débute en 2010 l'exploitation de ses ressources pétrolières. Les ressources pétrolières, comme les afflux d'aide, présentent des risques à travers l'expansion fiscale qu'elles permettent et le phénomène dit de « Dutch disease ».

Le modèle est proche à celui d'Adam et al. (2009) mais avec des différences notables, comme l'introduction d'un secteur bancaire et d'importantes frictions de marché. Une autre différence entre ces deux approches est que le gouvernement peut s'ajuster non seulement par ses différentes opérations sur les marchés monétaires et de titres, mais également en faisant varier les taux de taxation (supposés constants chez Adam et al. (2009)). Les simulations sont réalisées en tenant compte de la politique des autorités sur la manière dont ils dépenseront les revenus pétroliers à chaque période (lissage ou dépenses complètes) et aussi par rapport aux parts allouées aux secteurs non échangeable et aux investissements. La comparaison de ces différentes politiques permet aux auteurs d'analyser leurs impacts sur le comportement futur des composantes du cycle au Ghana.

Selon Dagher et al. (2010), l'augmentation des dépenses publiques consécutives à l'arrivée des ressources pétrolières n'entraînerait pas forcément une hausse de l'inflation par rapport à sa valeur courante si toutefois la composition courante de ces dépenses entre les secteurs échangeable et non échangeable restait non changée. A moyen terme, le comportement du taux de change pourrait jouer un rôle crucial dans le processus d'ajustement, son appréciation pourrait entraîner une baisse de la 
productivité due aux externalités des effets d'expérience dans le secteur non échangeable. Toutefois, ces effets négatifs pourraient se réduire avec une augmentation des dépenses publiques en investissement en capital. La politique monétaire consistant à réduire le taux d'inflation pourrait à court terme réduire la croissance. Les résultats montrent que les accumulations des réserves au moment où les dépenses publiques augmentent pourraient amplifier la pression sur la demande agrégée et générer des niveaux élevés d'inflation. Dagher et al. (2010) montrent également que les politiques limitant les frictions sur les marchés du travail et financiers pourraient avoir des effets bénéfiques sur toute l'économie, notamment pour accompagner les effets des chocs sur les revenus pétroliers.

\section{Bilan et perspectives}

Le projet scientifique de la littérature présentée dans cette revue était d'appliquer les modèles d'équilibre général dynamique et stochastique aux pays d'ASS. Ce projet a été au moins en partie réalisé avec succès. Les contributions de cette littérature apportent des réponses propres quant à l'origine de la plus grande instabilité de ces économies et développent des recommandations de politique économique tenant compte des spécificités de ces économies. Ces réponses et ces recommandations ne sont naturellement pas exemptes de critiques mais prouvent que ces modélisations apportent un éclairage original à ces questions. Quatre points nous semblent importants à discuter en conclusion pour évaluer les perspectives de cette littérature.

\subsection{La question des données}

La faible qualité des données est une question essentielle pour la poursuite de ce programme de recherche. Face à cette difficulté, deux stratégies ont été suivies. La première stratégie a consisté à recourir à des bases de données constituées de plusieurs pays afin de compenser la faible information pour chacun d'entre eux, c'est le cas chez Mendoza (1995), Koze et Reizman (2001), Adam et al (2009) et Özbilgin (2010). En faisant la moyenne des phénomènes observés sur des groupes de pays, cette stratégie permet d'asseoir les conclusions sur des comportements moyens plutôt que sur la situation d'une ou quelques économies en particulier. La seconde stratégie consiste à se restreindre aux pays pour lequel des séries de meilleure 
qualité et de plus haute fréquence sont disponibles. Cela permet par exemple à Peiris et Saxegaard (2010) de considérer des séries trimestrielles offrant une appréhension plus fine des phénomènes cycliques qu'avec des séries annuelles, mais par contre sur une échelle de temps très courte (un peu plus d'une dizaine d'années). Ces deux stratégies continueront certainement à être alternativement suivies selon le contexte pour exploiter au mieux des données macroéconomiques trop rares.

Au final, les travaux présentés dans cette revue ont montré que ces données, malgré leurs limites, permettent d'aborder une grande variété des aspects du cycle économiques à travers les principaux agrégats réels (production, consommation et investissement), la sphère nominale (inflation et taux d'intérêt) et les aspects internationaux (termes de l'échange, taux d'intérêt mondiaux et aide au développement). En comparaison aux études sur les pays développés, les variables qui font cruellement défaut sont celles du marché du travail. L'emploi est une variable cruciale du cycle économique qui a joué un rôle fondamental dans les développements récents de la théorie de cycle à travers les questions relatives à la mesure des chocs sur la productivité des facteurs et de leurs effets ; voir notamment Burnside et al. (1996) et Gali (1999). L'absence de séries longues d'emploi empêche d'emblée d'aborder ces questions dans les pays d'ASS alors qu'elles ont fait fortement progresser la recherche sur le cycle des pays développés.

Pour conclure sur ce point, soulignons que le problème de la qualité des données est une difficulté commune à toutes les études des pays d'ASS, qu'elles portent sur le cycle économique ou non. Par exemple, pour l'étude de la croissance économique, Young (2009) nous invite à utiliser avec prudence les séries macroéconomiques disponibles et à réviser profondément les mesures traditionnelles de la croissance dans ces pays.

\subsection{Modélisation VAR et DSGE}

Parallèlement aux modèles DSGE, les modèles économétriques Vectoriel AutoRegressif (VAR) structurels ont été appliqués aux pays d'ASS. Ces modèles permettent également, à partir d'hypothèses structurelles, d'identifier la contribution des différents types de chocs aux fluctuations économiques. Par exemple Hoffmaister et al. (1998) identifie par un modèle VAR structurel la contribution respective des chocs extérieurs et des chocs domestiques aux fluctuations de la 
production et compare les résultats selon l'appartenance ou non des pays à la zone franc CFA. Les modèles VAR structurels sont également utilisés précisément pour valider la pertinence des modèles DSGE proposés pour les pays en développement (voir par exemple Kose et Riezman, 2001).

L'apport décisif des modèles DSGE est l'évaluation des politiques économiques qu'ils permettent. Contrairement aux modèles VAR structurels, ils ne permettent pas seulement de proposer une analyse des fluctuations réalisées, ils peuvent également servir de base à l'étude de scénarios de politique économique. Cet apport explique en partie leur succès dans l'analyse macroéconomique actuelle. II convient également d'indiquer que ce succès découle des bonnes propriétés empiriques des modèles DSGE mises en évidence pour les économies développées. Pour l'économie de la zone euro, Smets et Wouters (2003) ont montré qu'un modèle DSGE pouvait mieux prédire les fluctuations qu'un modèle VAR. Ce résultat remarquable a donné lieu à une importante littérature montrant la pertinence empirique des DSGE même lorsqu'ils sont comparés à des modèles VAR; voir Collard et Fève (2007). Montrer que cette conclusion s'applique également aux pays d'ASS apporterait certainement une légitimité supplémentaire aux applications des modèles DSGE à ces pays.

\subsection{Vers davantage de mécanismes spécifiques d'amplification et de propagation}

La compréhension des mécanismes d'amplification et de propagation des chocs spécifiques aux pays d'ASS nous semblent une voie importante à explorer pour cette littérature. Le fait de recourir presque systématiquement au modèle de petite économie ouverte est un point fort de cette littérature par l'homogénéité du cadre d'analyse que cela a permis. Dans ce cadre d'analyse, l'essentiel des travaux a porté sur les sources d'impulsion spécifiques des pays d'ASS avec peu d'adaptation du modèle théorique à ces économies à l'exception de la modélisation de la participation limitée aux marchés financiers par Özbilgin (2010) et de la politique monétaire par Adam et al (2009).

Du point de vue méthodologique, mettre l'accent sur les sources d'impulsion a incontestablement facilité le développement de cette littérature en plaçant l'hétérogénéité sur la partie la plus aisément mesurable de la modélisation et non sur la structure des pays qui peut varier très fortement d'un pays à l'autre. Néanmoins, 
fort des résultats obtenus sur les sources d'impulsion, il nous semble que ces modèles pourraient s'enrichir de mécanismes spécifiques dont on serait en mesure d'évaluer la pertinence compte tenu des résultats déjà connus de cette littérature. $A$ titre d'exemple, la répartition des ressources entre les secteurs formels et informels et les migrations régionales entre zones urbaines et rurales sont reconnues comme des éléments cruciaux en économie du développement et pourraient également jouer un rôle dans le processus de transmission des chocs à l'économie. Cette perspective de recherche s'inscrirait aussi dans une volonté de mieux intégrer les analyses du court terme et du long terme.

\subsection{Un retour à l'objectif initial d'une analyse intégrée de la croissance et du cycle}

L'objectif initial du programme de recherche de la théorie des cycles réels était de réconcilier l'analyse du court et du long terme séparée durant plusieurs décennies au sein de l'analyse économique. Cooley et Prescott (1995) retracent cette évolution et insistent sur le fait que dans la théorie moderne du cycle d'activité, « la croissance et les fluctuations ne sont pas des phénomènes distincts qui doivent être étudiés avec des données distinctes et des outils analytiques différents ${ }^{19}$. Cette volonté, formulée à l'origine pour les économies les plus développées, trouve pleinement son sens pour les pays d'ASS qui souffrent simultanément d'une croissance trop faible et d'une trop grande instabilité de leur activité économique. Dans ce contexte, il faut concevoir des recommandations de politique économique intégrant ces deux objectifs de croissance et de stabilité. Les modèles DSGE présentés dans cette revue de la littérature constituent des points de départ naturels puisqu'ils se fondent sur les modèles d'équilibre général dynamique (et déterministe) utilisés pour l'évaluation des politiques de croissance. Leur capacité à rendre compte du cycle économique en ASS mise en évidence dans cette revue ouvre la perspective de l'évaluation conjointe des politiques de croissance et de stabilisation.

\footnotetext{
19 "Modern business cycle theory starts with the view that growth and fluctuations are not distinct phenomena to be studied with separate data and different analytical tools" Cooley et Prescott (1995) p. 4.
} 


\section{Bibliographie}

Acemoglu D. 2010.Theory, general equilibrium and political economy in development economics.Working paper, http://www.nber.org/papers/w15944.

Adam C., O'Connell S., Buffie E., Pattillo C., 2009. Monetary rules for managing aid surges in Africa. Review of Development Economics, 13(3), 469.

Adolfson, M., Laséen, S., Lindé, J., Mattias, V., 2008. "Evaluating an estimated new Keynesian small open economy model," Journal of Economic Dynamics and Control, 32(8), 2690-2721.

An, S., Schorfheide, F., 2007. Bayesian Analysis of DSGE Models, Econometric Reviews, 26(2-4), 113-172.

Arellano C., Bulir A., Lane T., Lipschitz, L., 2009. The dynamic implication of foreign aid its variability. Journal of Development Economics 88, 102.

Arellano C., Mendoza, E. G., 2002. Credit friction and sudden stop in small open economies: An equilibrium business cycle framework of Emerging Markets crises. NBER working paper $n^{\circ} 8880$.

Bénassy, J.-P., 2008. Dynamic models with non-clearing markets, the New Palgrave Dictionary of Economics, second edition, Macmillan.

Berg, A., Gottschalk, J., Portillo, R., Zanna, L., 2010. The Macroeconomics of Medium-Term Aid Scaling-Up Scenarios.IMF Working Paper.

Burnside, A. C., Eichembaum, M. S., Rebelo, S. T., 1996 Sectoral Solow residual. European Economic Review, 40(3-5), 816-869.

Buffie, E., Adam, C., O'Connell, S., Pattillo, C., 2008.Riding the wave: Monetary responses to aid surges in low-income countries, European Economic Review52(8), pages 1378-1395.

Bulir, A., Hamann, J. A., 2007. Volatility of development aid: From the frying pan into the fire? World Development, 36(10), 2048-2066.

Christiano, L. J., Eichenbaum, M, Evans, C. L., 2005. Nominal rigidities and the dynamic effects of a shock to monetary policy. Journal of Political Economy 113 (1), 1-45.

Clarida, R., Gali, J., Gertler, M., 1999. The Science of Monetary Policy: A New Keynesian Perspective, Journal of Economic Literature, 37(4), 1661-1707 
Collier, P., Gunning, J. W., 1999. Why has Africa grown slowly? Journal of Economic Perspective, 13(3),3-22.

Collard, F, Fève, P. 2007. Les modèles VAR ou DGSE: Que choisir ? ", Économie et Prévision, n9183, mai 2007, p. 153-174.

Cooley, F., Prescott, E. C., 1995.Economic Growth and Business Cycles.Princeton University Press.

Dagher J., Gottschalk, J., 2010. Oil windfalls in Ghana: a DSGE approach. IMF WP/10/116.

Danthine, J. P., Donaldson, J. B., 1993. Methodological and empirical issues in real business cycle theory. European Economic Review, 37 (1).

Easterly, W., 2003. Can Foreign Aid by Growth? The Journal of Economic Perspectives, 17(3),23-48(26).

Gali, J. 1999 Technology, Employment and Business Cycle: Do Technology Shocks Explain Aggregate Fluctuations? American EconomicReview, 89, 249-271.

Hairault, J.-O., 1999, Vers une nouvelle synthèse néoclassique? La théorie des cycles réels n'est pas ce que l'on croit, Revue d'Economie Politique, 109, 613670.

Hoffmaister, A. W., Roldos, J. E., Wickham, P., 1998. Macroeconomic Fluctuations in Sub-Saharan Africa, IMF Staff Papers, 45(1), pages 132-160.

Houssa R., RaduPuslenghea. 2010. A model for monetary policy analysis for SubSaharan Africa. Open Econ Review, 21, 127-145.

Kose, M. A., 2002. Explaining business cycles in small open economies, how much do world price matter? Journal of International Economics, 56, 299-327.

Kose, N. A., Riezman, R., 2001. Trade shocks and macroeconomic fluctuation in Africa. Journal of Development Economic, 65(1), 55-80.

Kravis, B., Henston, A., Summers, R., 1982. Word product and Income: International comparisons of Real Gross Product. Johns Hopkins University Press.

Kydlland, F., Prescot, E., 1977. Rules Rather than Discretion: the inconsistency of Optimal Planes. Journal of Political Economy, 85, 473-491.

Kydlland, F., Prescot, E., 1982.Time to Build and Aggregate Fluctuations.Econometrica, 50, 1345-1970. 
Loayza, N.V., Ranciere, R., Serven, L., Ventura, J., 2007. Macroeconomic volatility and welfare in developing countries: and introduction. The world Bank Economic Review,21, 343-357.

Lucas, R. E., 1976. Econometric Policy Evaluation: a Critique in K. Bruner et A.Melizer. ed. The Phillips Curve and Labor Markets. Carnegie Rochester Conference Series in Public Policy. 1, 19-46. Amsterdam-North Holland.

Lucas, R. E., 1987. Models of Business Cycles, Blackwell, Oxford.

Mendoza E. G.,1995. The terms of trade, the real exchange rate, and economic fluctuations. International Economic Review, 36(1), 101-137.

Ostry, J, D., 1990. The balance of trade, Terms of Trade, and Real Exchange Rate: An Intertemporal optimizing Framework. IMF Staff Papers 35(1998), 541-573.

Özbilgin H., 2010. Financial market participation and the developing country business cycle. Journal of Development Economics 92, 125-137.

Pallage, S., Robe, M., 2003. The Welfare cost of Economic Fluctuations in Developing Countries. International Economic Review, 44(2), 677-698.

Peiris S., Saxegaard, M., 2010. An Estimated Dynamic Stochastic General Equilibrium Model for Monetary Policy Analysis in Mozambique. IMF staff papers, vol. 57, n9, pp. 256-280."

Rajan, R.G., Subramanian, A., 2008. Aid and Growth: What Does the Cross-Country Evidence Really Show?, The Review of Economics and Statistics, 90(4), 643665.

Ramey, G., Ramey, V.A., 1995. Cross-Country Evidence on the Link Between Volatility and Growth. American Economic Review, 85, p1138-1151.

Rand, J., Tarp, F., 2002. Business Cycles in Developing Countries: Are they Different? World Development, Elsevier,30, 2071-2088.

Smets, F., Wouters, R., 2007. Shocks and Frictions in US Business Cycles: A Bayesian DSGE Approach, American Economic Review, 97(3), 586-606.

Stadler, G.W., 1994. Real Business Cycles, Journal of Economic Literature, XXXII, 1750-1783.

Stockman, A., Tesar, L., 1990.Taste and Technology in a tow-country model of the Business Cycle Explaining International Comovements.American Economic Review. 
Turnovsky, S. J., Chattopadhyay, P., 2003. Volatility and growth in developing economies: somme numerical results and empirical evidence. Journal of International Economics, 59(2), 267-295.

Woodford, M., 2003.Foundations of a Theory of Monetary Policy. Princeton University Press, 1, XV-785P.

Word Bank, 1998. Assessing Aid. What Works, What Doesn't, and Why. Oxford University Press.

Young., 2009. The African growth miracle, mimeo LSE. 


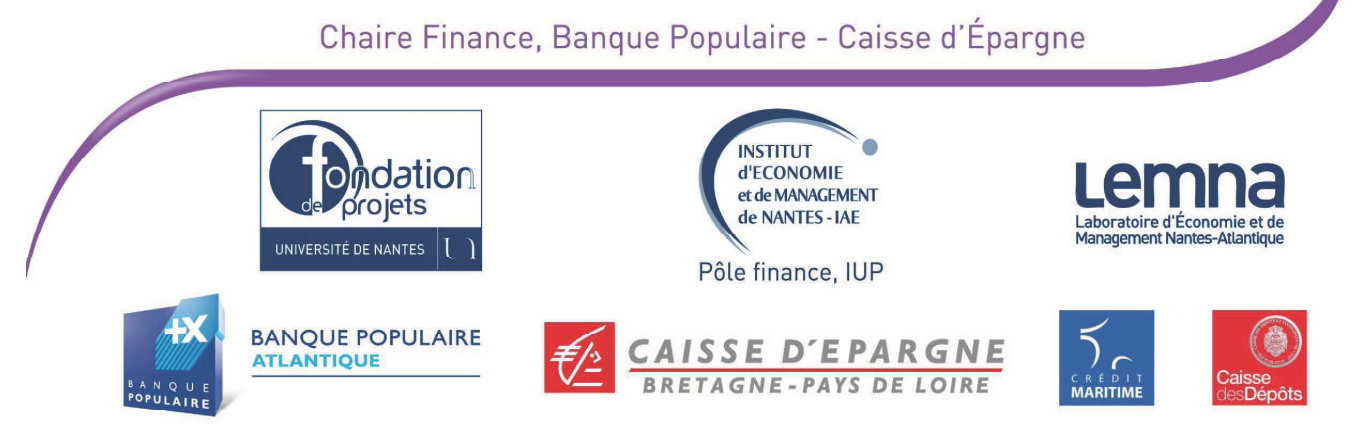

Les Cahiers de la Chaire Finance rassemblent les documents de travail du LEMNA s'inscrivant dans des projets de recherche qu'elle soutient.

$\mathbf{N}^{\circ}$ 2012-06 Are Islamic Indexes more Volatile than Conventional Indexes? Evidence from Dow Jones Indexes

CHARLES Amélie, DARNÉ Olivier et POP Adrian

$\mathbf{N}^{\circ}$ 2012-07 Large Shocks in the Volatility of the Dow Jones Industrial Average Index: 1928-2010

CHARLES Amélie et DARNÉ Olivier

$\mathbf{N}^{\circ}$ 2012-08 The Quality of Private Monitoring in European Banking: Completing the Picture

POP Adrian et POP Diana

$N^{\circ}$ 2012-09 Effets socioéconomiques de la crise financière : implications pour le Vietnam

LAUZANAS Jean-Marc, PERRAUDEAU Yves et POP Adrian

$N^{\circ}$ 2012-31 Efficiency Gains from Narrowing Banks: A Search-Theoretic Approach TRIPIER Fabien

$N^{\circ}$ 2012-32 Volatility Persistence in Crude Oil Markets

CHARLES Amélie et DARNÉ Olivier

$N^{\circ}$ 2012-40 La modélisation en équilibre général et stochastique des cycles économiques en Afrique Sub-saharienne : une revue de la littérature NOUASSI Claude Francis et TRIPIER Fabien

Les opinions exposées dans ce document n'engagent que les auteurs. Ceux-ci assument la responsabilité de toute erreur ou omission.

La Chaire Finance est une initiative de la Banque Populaire Atlantique, la Caisse d'Epargne Bretagne-Pays de Loire, la Caisse des Dépôts, du Crédit Maritime et de l'Université de Nantes, sous l'égide de la Fondation de Projets de I'Université de Nantes.

Site web : http://www.univ-nantes.fr/fondation/chairefinance

\section{Contact}

Chaire Finance, Banque Populaire - Caisse d'Epargne

IEMN-IAE, Chemin de Censive du Tertre - BP 52231

44322 Nantes cedex 3

Tél : +33(0)2 40141660

Fax : +33 (0)2 40141650 